\title{
Capteur SAW implantable dédié à la télémesure de la température et de la pression artérielle : le projet ANR-TECSAN CIMPA
}

\author{
Implantable SAW sensor for telemetry of temperature and blood pressure: \\ The ANR-TECSAN CIMPA project
}

\author{
S. Tourette ${ }^{\mathrm{a}, *}$, L. Chommeloux ${ }^{\text {a }}$, J.F. Le Guen ${ }^{\mathrm{a}}$, J.M. Friedt ${ }^{\mathrm{a}}$, P. Ménage ${ }^{\mathrm{a}}$,

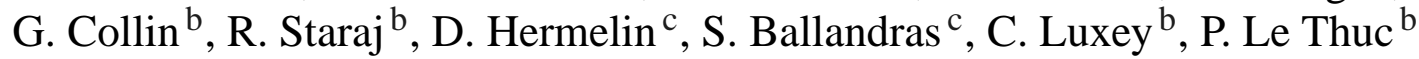 \\ a SENSeOR, parc du «Font-de-l'Orme», lot n³, 694, avenue du Docteur-Maurice-Donat, 06250 Mougins, France \\ b Laboratoire d'électronique, antennes et télécommunications, université de Nice-Sophia Antipolis, \\ CNRS, 250, rue Albert-Einstein, bâtiment 4, 06560 Valbonne, France \\ ${ }^{\mathrm{c}}$ FEMTO-ST, département temps fréquence, 32, avenue de l'Observatoire, 25044 Besançon, France
}

\section{Résumé}

Le projet CIMPA (2007-2008), réalisé dans le cadre de l'appel ANR-TECSAN, a été coordonné par SENSeOR, en partenariat avec le LEAT et le FEMTO. Son objectif était la mise au point d'un démonstrateur de capteur surface acoustic waves (SAW) passif, implantable, permettant de mesurer la pression artérielle ainsi que la température. Ce capteur utilise la technologie des ondes acoustiques de surface (SAW) et est composé de trois résonateurs sur quartz. Un premier résonateur pour la mesure de température $(\mathrm{T})$, un second résonateur pour la mesure de la pression sanguine $(\mathrm{P})$ et un troisième résonateur qui sert de référence $(\mathrm{R})$. Ce capteur est compatible des exigences d'encombrement inhérentes à une implantation dans une artère puisque la taille du résonateur avec ses antennes, dans sa configuration la plus compacte, est de l'ordre $5,2 \mathrm{~mm} \times 3,7 \mathrm{~mm} \times 0,85 \mathrm{~mm}$. Il ne nécessite aucune source d'énergie embarquée et peut être interrogé via une liaison radiofréquence (RF) grâce à un interrogateur qui émet un signal dans la bande industriel, scientifique et médical (ISM). Le capteur sollicité par le signal d'interrogation, via son antenne intégrée de très petite dimension, émet à son tour un signal qui porte les informations de pression et de température vues par ce dernier. L'interrogateur capte alors le signal émis par le capteur et en extrait, via un traitement du signal adapté, les informations de pression et de température. La gamme de mesure de pression s'étend de 1 bar à 1,35 bar avec une précision de l'ordre de $2 \%$.

Mots clés : Capteur de pression et de température ; Résonateurs à ondes acoustiques de surface ; Sans-fil ; Passif ; Implantable ; Antenne spirale

\section{Abstract}

The CIMPA Project (2007-2008), led under the ANR-TECSAN Call, was coordinated by SENSeOR, in partnership with the LEAT and the FEMTO. The aim of the project was to develop a surface acoustic wave sensor demonstrator, passive implantable to monitoring the blood pressure and the temperature. This sensor uses the surface acoustic wave technology (SAW) and is composed of three resonators using quartz as piezoelectric substrate. One resonator for temperature measurement $(\mathrm{T})$, a second resonator for the blood pressure measurement $(\mathrm{P})$ and a third resonator, which is used as reference $(\mathrm{R})$. This sensor is compatible with size requirements inherent to an implantation in an artery, since the size of the resonator with its antennas in its most compact configuration is of the order of $5.2 \mathrm{~mm} \times 3.7 \mathrm{~mm} \times 0.85 \mathrm{~mm}$. It requires no embarked sources of energy and being able to be interrogated by a radiofréquence (RF) connection thanks to a transceiver, which emits a signal in the band industrial, scientific and medical (ISM). The sensor (connected to an integrated antenna on quartz of very small dimension) requested by the signal of interrogation

\footnotetext{
* Auteur correspondant.

Adresse e-mail : stephane.tourette@unice.fr (S. Tourette).
} 
emits in its turn a signal, which carries information of pressure and temperature. The transceiver then collects apart from its transmitting phase the signal emitted by the sensor and extracts by an appropriate signal processing method information of pressure and temperature. The targeted range for pressure measurement extends from 1 bar to 1.35 bar with an accuracy of about $2 \%$ FS.

Keywords: Pressure and temperature sensor; Surface acoustic wave resonator; Wireless; Passive; Implantable; Spiral antenna

\section{Introduction}

Les capteurs sans-fil utilisant la technologie des ondes acoustiques de surface (SAW) demeurent en pleine expansion depuis plusieurs années dans de nombreuses applications domestiques, médicales, industrielles ou militaires [1,2]. Leur principal avantage réside dans son fonctionnement passif, c'està-dire sans batterie. Il serait donc possible d'implanter ces capteurs SAW dans un corps humain afin de permettre une surveillance médicale. Ce dispositif permettrait ainsi d'améliorer la santé, le diagnostic, le suivi et par conséquent le confort de patients souffrant d'hypertension par exemple. En effet, il s'agit de répondre aux besoins de situations cliniques pour lesquelles la mesure en continu de la pression sanguine permettrait d'effectuer des diagnostics précis, des contrôles et des adaptations en temps réel de traitement d'hypertension ainsi que des alarmes en cas de problèmes. Cette mesure en continu serait possible sans la nécessité d'une intervention chirurgicale supplémentaire et pourrait être utilisée pour un suivi à domicile, permettant ainsi une réduction du temps d'hospitalisation.

\section{Conception d'un capteur des ondes acoustiques de surface à $2,45 \mathrm{GHz}$}

Le concept initial du capteur repose sur une seule puce à trois niveaux, enrobée par un matériau biologiquement inerte. Deux coupes de quartz utilisées $\mathrm{C} 1$ et $\mathrm{C} 2$ offrant des caractéristiques appropriées en termes de dérive thermique et de sensibilité aux contraintes (satisfaisant le compromis précision sur estimation de la température et de la pression/occupation de la bande ISM à $2,45 \mathrm{GHz}$ ) ont été identifiées grâce à l'utilisation de codes de calcul développés par l'institut Femto [3]. Les Fig. 1 et 2 présentent le schéma de principe du capteur de pression et de

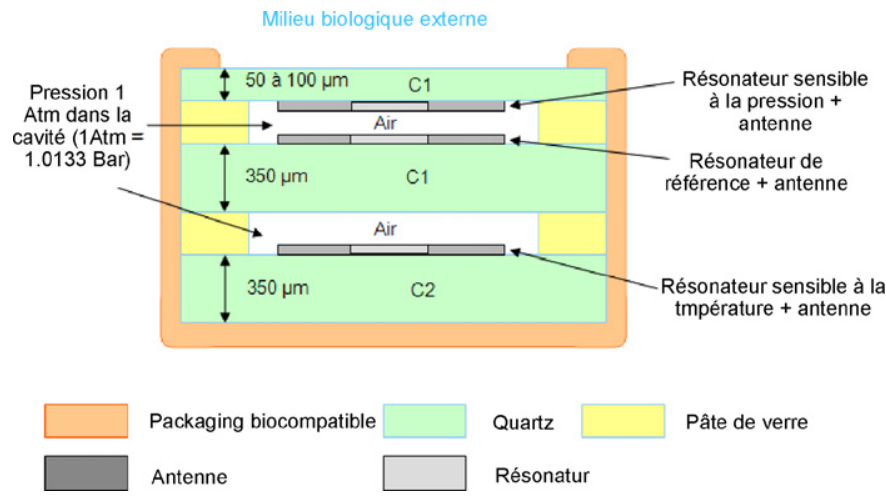

Fig. 1. Schéma de principe du dispositif in vivo (vue en coupe dans le plan vertical). température (composé de trois résonateurs) ainsi que l'allure d'un des trois résonateurs muni de leur antenne à $2,45 \mathrm{GHz}$.

Le premier résonateur (utilisant une coupe de quartz $\mathrm{C} 1$ ) se situe sur la partie supérieure amincie (50 à $100 \mu \mathrm{m})$ sensible à la pression (résonateur type $\mathrm{P}$ ). Le second résonateur au milieu de la structure, utilisant la même coupe de quartz $\mathrm{C}$ 1, est positionné sur une partie non amincie, dans une zone où les contraintes sont considérées négligeables (résonateur de référence $\mathrm{R}$ ). Le troisième résonateur STW (zone inférieure du capteur) est également localisé sur un substrat non aminci et utilise une coupe de quartz $\mathrm{C} 2$ choisie de façon à présenter des caractéristiques de dérive en température satisfaisant le compromis précision sur estimation de la température/occupation de la bande ISM à 2,45 GHz (résonateur type T).

\section{Conception de l'antenne associée au capteur des ondes acoustiques de surface à $2,45 \mathrm{GHz}$}

Des concepts d'antennes intégrées sur le substrat de quartz ont été étudiés par le LEAT (dipôles méandres répondant aux exigences d'encombrement) [4]. Les performances de ces antennes ont été optimisées en tenant compte de la nature du dépôt métallique utilisée pour la réalisation du dispositif SAW, de la géométrie des empilements de tranche (Fig. 1), des caractéristiques électromagnétiques du quartz et du milieu biologique environnant. Il a été montré que l'utilisation originale de brins

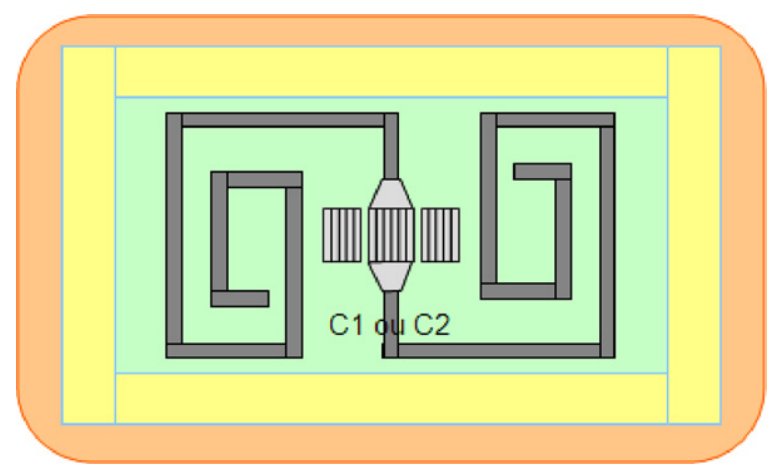

Fig. 2. Schéma de principe d'un résonateur et de son antenne vue de dessus (coupe dans le plan horizontal).

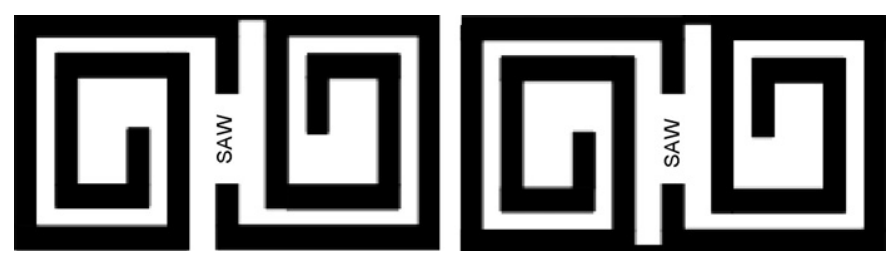

Fig. 3. Exemple de structures d'antennes simulées avec (à gauche) un brin de court circuit et (à droite) deux brins de court-circuit. 


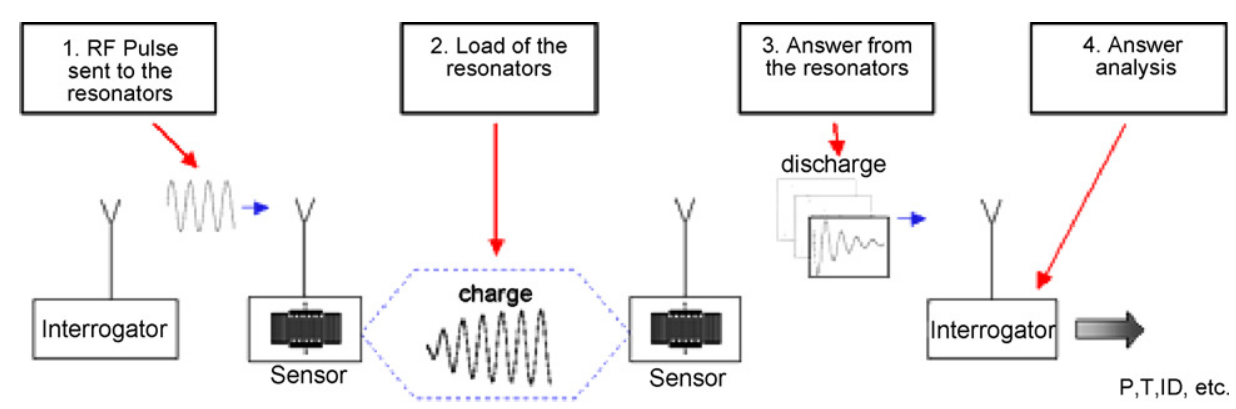

Fig. 4. Principe d'interrogation sans fil d'un capteur SAW constitué d'un résonateur unique.

de court-circuit (Fig. 3) de largeur, longueur, et de localisation variables permettait d'adapter l'impédance de l'antenne à celle du résonateur et d'optimiser son gain (qui demeure néanmoins faible du fait de l'environnement biologique). Il a par ailleurs été montré que les performances de l'antenne seraient très sensibles aux variations environnementales biologiques (d'un individu à l'autre par exemple).

Deux types de capteurs ont été conçus (correspondant à deux tailles de diaphragme) présentant des encombrements respectifs (typiques) de $5,2 \times 3,7 \times 0,85 \mathrm{~mm}^{3}$ et de $8,2 \times 5,6 \times 0,85 \mathrm{~mm}^{3}$.

\section{Principe d'interrogation}

SENSeOR développe des capteurs à ondes acoustiques de surface pour la mesure de température, de pression et de contraintes. Ces capteurs purement passifs présentent la particularité de pouvoir être interrogés via une liaison radiofréquence (RF) sans fil sur des distances pouvant varier (selon le milieu de propagation et la fréquence utilisée) de quelques centimètres à plusieurs mètres. L'apport d'énergie est effectué par un interrogateur qui remplit le rôle d'émetteur et de récepteur. Le principe d'interrogation d'un capteur SAW s'apparente aux techniques Radar. Il est rappelé sur la Fig. 4 ci-dessous, pour une configuration à base de capteurs résonants.

Le système complet se compose d'une unité d'interrogation (constituée elle-même d'une partie émetteur et d'une partie récepteur) et du capteur de pression SAW. Le système d'interrogation ainsi que le capteur SAW sont munis d'une antenne adaptée à la bande de fréquence de travail (bande ISM centrée autour de $2,45 \mathrm{GHz}$ ) qui permet d'effectuer une interrogation sans fil du capteur. Le mode d'interrogation est le suivant :

- l'émetteur du système d'interrogation envoie un signal d'interrogation vers le capteur SAW à une fréquence proche de la fréquence de résonance du capteur;

- le signal électromagnétique reçu par l'antenne du capteur est converti en ondes de surface via un transducteur à peignes inter-digités grâce aux propriétés piézolectriques du substrat utilisé ;

- ces ondes de surface voient leurs propriétés modifiées en fonction de la pression et de la température qui affectent les conditions de propagation (en particulier la vitesse de phase qui conditionne directement la fréquence de résonance);
- le capteur réémet un écho à sa fréquence de résonance qui porte donc l'information liée au phénomène de pression;

- le récepteur du système d'interrogation détecte en dehors de la plage temporelle d'émission tout ou partie de l'écho du capteur SAW et extrait de la réponse reçue l'information de pression recherchée.

\section{Bilan de liaison dans le cas de l'interrogation d'un capteur implanté à $2,45 \mathrm{GHz}$}

Il était également nécessaire de déterminer si le niveau de puissance typique reçu (tout en conservant une puissance d'émission compatible des normes ISM) par l'interrogateur dans le cas où le capteur est enfoui dans un milieu biologique était compatible avec la sensibilité de détection. Un modèle simplifié directement dérivé de l'équation du RADAR a donc été mis au point. Ce modèle permet, à partir de la connaissance de la puissance émise, des distances parcourues dans l'air, dans le milieu biologique, des pertes aux interfaces, du gain des antennes du capteur et de l'interrogateur de déterminer le niveau de puissance reçue par l'interrogateur. La Fig. 5 montre un exemple de bilan de liaison (calculé sur la base du modèle précité) dans lequel le capteur se situe sous $2 \mathrm{~cm}$ de muscle (Fig. 6).

La distance interrogateur/patient est de $1 \mathrm{~m}$. On constate en particulier qu'à $2,45 \mathrm{GHz}$ l'atténuation liée à la propagation dans l'air est de l'ordre de $28 \mathrm{~dB}$ soit $2 \times 28 \mathrm{~dB}=56 \mathrm{~dB}$ sur un aller-retour. Si l'on ajoute les pertes liées à la désadaptation dues aux interfaces ainsi que les pertes de propagation dans le milieu biologique, on constate que le niveau de signal reçu par l'interrogateur est de l'ordre de $-121 \mathrm{dBm}$ soit $50 \mathrm{~dB}$ sous la limite de détection $(-70 \mathrm{dBm})$ de l'interrogateur.

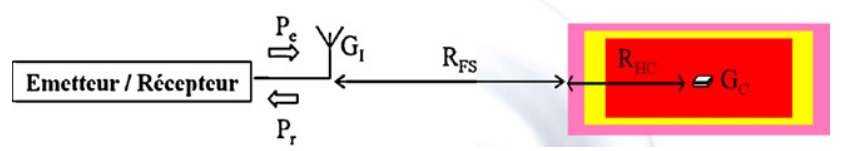

Fig. 5. Modèle utilisé pour le calcul du bilan de liaison simplifié. $\mathrm{P}_{\mathrm{e}}$ : puissance émise $; \mathrm{G}_{\mathrm{I}}, \mathrm{G}_{\mathrm{C}}$ : gains des antennes interrogateur et capteur ; $\mathrm{R}_{\mathrm{FS}}$ : distance parcourue dans l'air; $\mathrm{R}_{\mathrm{HC}}$ : distance parcourue dans le milieu biologique. 


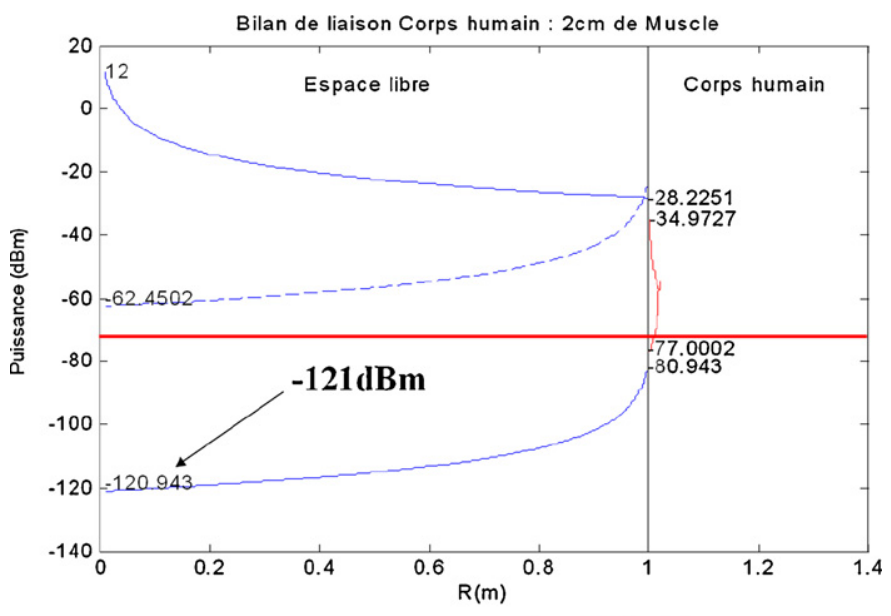

Sensibilité interrogateur

Fig. 6. Exemple de bilan de liaison simplifié pour un capteur implanté sous $2 \mathrm{~cm}$ de muscle dans le cas ou l'interrogateur est situé à $1 \mathrm{~m}$ du patient. La courbe en pointillés correspond au bilan de liaison ne tenant compte que de la propagation dans l'air $(2 \mathrm{~m} \times 1 \mathrm{~m})$.

\section{Fabrication et caractérisation des démonstrateurs à $2,45 \mathrm{GHz}$}

La fabrication des démonstrateurs a nécessité l'enchaînement des étapes suivantes (notons que les quatre dernières étapes de fabrication ont nécessité le développement des briques technologiques associées dans le cadre de ce projet) : fabrication des dispositifs SAW et caractérisation sous pointes, dépôt de pâte de verre par sérigraphie, scellement des tranches, amincissement et caractérisation électrique, enrobage biocompatible.

La caractérisation sous pointes nous a permis de constater que les structures résonantes synchrones ont des performances très sensibles aux dispersions technologiques contrairement aux structures asynchrones qui semblent être un meilleur choix pour la fabrication de résonateurs STW sur quartz à $2,45 \mathrm{GHz}$. L'ensemble des procédés de fabrication a fait l'objet d'une recherche de sous-traitance et des fabrications pilotes ont été engagées dans le cadre de tranches de quartz vierges dans un premier temps puis avec des tranches portant des dispositifs SAW. La Fig. 7 ci-dessous présente le résultat d'essais de scellement verre entre deux wafers vierges (sans dispositifs SAW). Les zones sombres correspondent à la pâte de verre, chaque petit rectangle correspond à une cavité hermétique qui peut être isolée après une opération de découpe. Les essais de scellement avec deux tranches de coupe différente $\mathrm{C} 1$ et $\mathrm{C} 2$ ont montré que la différence de coefficient d'expansion thermique pouvait engendrer des problèmes de fissure, voire de rupture des tranches après scellement ce qui nous a conduit à sceller séparément les tranches de coupe différente et donc à abandonner le concept de dispositif à trois niveaux (Fig. 1) pour deux structures à deux niveaux.

L'étape suivante (pour la partie mesure de la pression) consiste à amincir le substrat de quartz à des épaisseurs pouvant typiquement comprises entre 50 et $100 \mu \mathrm{m}$. La Fig. 8 ci-dessous montre le résultat d'un essai d'amincissement pleine plaque sur un ensemble de deux tranches scellées. Dans ce cas, l'épaisseur

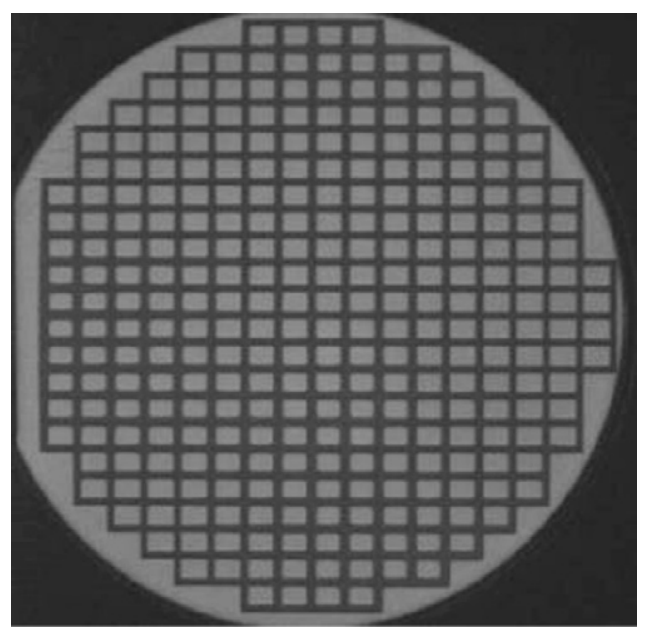

Fig. 7. Ensemble de deux wafers de quartz vierge associés par un procédé de scellement verre.

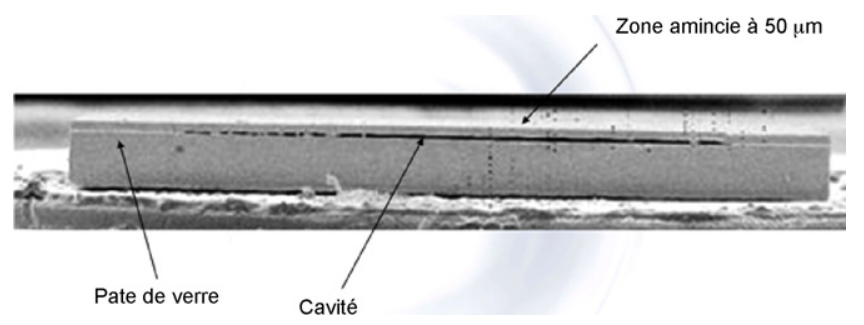

Fig. 8. Ensemble de deux wafers vierges amincis pleine plaque.

d'un des deux wafers a été réduite à $50 \mu \mathrm{m}$ (l'épaisseur initiale du wafer étant de $350 \mu \mathrm{m})$.

Nous avons constaté que les contraintes mécaniques des empilements généraient des déformations qui engendraient un mauvais rendement de l'opération d'amincissement. Nous avons mis en évidence le fait que l'amincissement après découpe (parmi les procédés testés) permet d'améliorer significativement le rendement de l'opération. Des tests en température en filaire

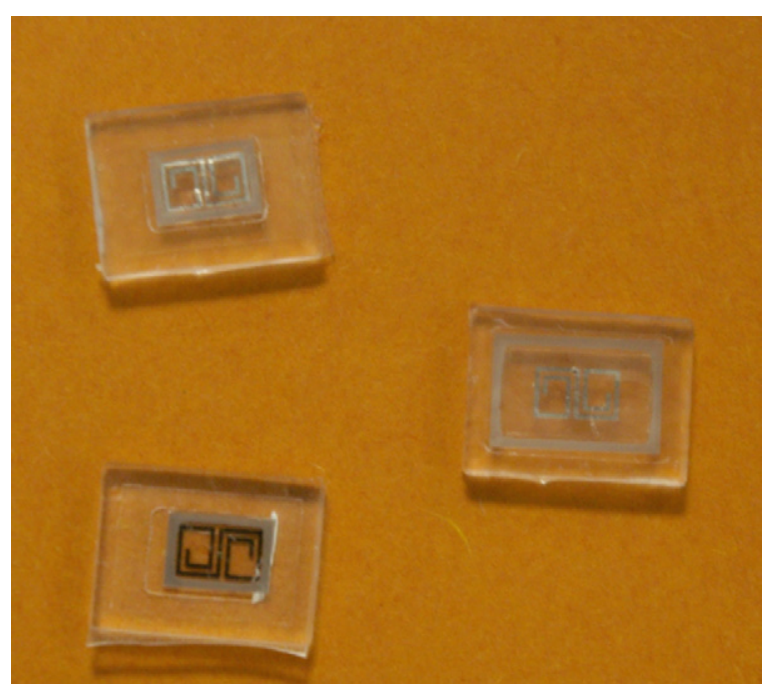

Fig. 9. Essai de moulage dans résine silicone d'un capteur prototype à base de résonateurs $\mathrm{SAW}$ à $2,45 \mathrm{GHz}$ avec antenne intégrée. 

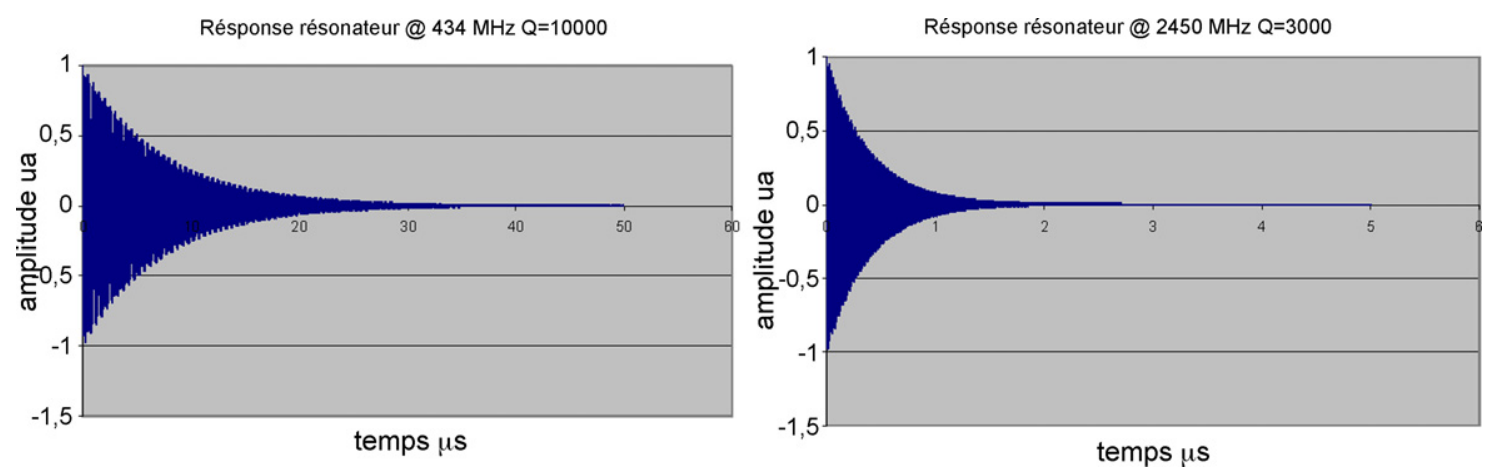

Fig. 10. Comparaison réponse résonateur à $434 \mathrm{MHz}(\mathrm{Q}=10000)$ et à $2,45 \mathrm{GHz} \mathrm{Q}=3000$.

sur des dispositifs de test de type $\mathrm{R}$ et $\mathrm{T}$ ont montré un très bon accord entre performances simulées et mesurées. Les essais de mesure de pression (dans une colonne d'eau) effectués en mode filaire sur des dispositifs de test type $\mathrm{P}$ ont montré que la fréquence de résonance était extrêmement sensible aux éléments parasites associés à l'environnement de mesure (capacité parasite liée à la présence du milieu aqueux). Cette sensibilité de la fréquence de résonance masque la variation de fréquence liée à la variation de pression. Finalement les essais d'enrobage biocompatible (par moulage) nous ont permis d'identifier les résines silicone permettant d'avoir en particulier une adhérence acceptable de la résine sur le quartz. Des dispositifs (R/P et T) avec antenne intégrée enrobés dans une résine biocompatible ont ainsi été réalisés (Fig. 9).

\section{Développement de l'unité d'interrogation à $2,45 \mathrm{GHz}$}

Un interrogateur prototype fonctionnant à $2,45 \mathrm{GHz}$ a été développé par le Femto sur la base de l'interrogateur à $434 \mathrm{MHz}$ moyennant certaines modifications, notamment au niveau de l'étage d'émission. La synthèse de la fréquence initialement effectuée sur la base d'un mélange a été revue au profit d'une solution à base d'un VCO (oscillateur commandé en tension) piloté par une PLL (boucle à verrouillage de phase). Cette solution permet en particulier de s'affranchir du filtre sur la voie d'émission (pour éliminer les harmoniques dues au mélange). Une validation préliminaire de cette modification d'architecture a été effectuée à $434 \mathrm{MHz}$. La voie de réception a également été caractérisée via des essais d'interrogation de résonateurs à
2,45 GHz. Il s'avère que la sensibilité (niveau minimum de signal détectable) est actuellement incompatible avec l'application développée. Cela s'explique par une mauvaise isolation entre voies d'émission et de réception et par le fait que le niveau de signal réémis par un résonateur à $2,45 \mathrm{GHz}(\mathrm{Q}=3000)$ s'atténue trop rapidement au cours du temps par rapport à un résonateur à $434 \mathrm{MHz}(\mathrm{Q}=12000)$. Ces éléments limitent actuellement la distance d'interrogation à une dizaine de centimètres dans l'air.

On voit, à titre d'exemple, sur la Fig. 10 que dans le cas d'un résonateur à $434 \mathrm{MHz}(\mathrm{Q}=10000)$, la réponse du résonateur est observable sur environ $35 \mu$ s alors que celle d'un résonateur à $2,45 \mathrm{GHz}$ ne l'est que sur une durée inférieure $2 \mu \mathrm{s}$. Pour des raisons de synchronisation entre étages d'émission et de réception, les premières périodes du signal réémis par le capteur ne peuvent être exploitées pour la détection. Si cela ne pose pas de problème à $434 \mathrm{MHz}$ compte tenu de la longueur temporelle de la réponse du capteur, cela s' avère rédhibitoire à $2,45 \mathrm{GHz}$ pour des résonateurs ne présentant des coefficients de qualité que de quelques milliers.

\section{Résultats de mesure à l'aide d'un capteur de pression à $434 \mathrm{MHz}$ non implantable}

Une étude a montré la possibilité de mesurer la température corporelle d'un chien à l'aide d'un résonateur SAW [5]. Parallèlement au développement du capteur à $2,45 \mathrm{GHz}$, un capteur à $434 \mathrm{MHz}$ utilisant un résonateur à ondes de Rayleigh a donc été conçu et fabriqué [6]. Une tranche de quartz sur laquelle on a au préalable réalisé un résonateur est scellée
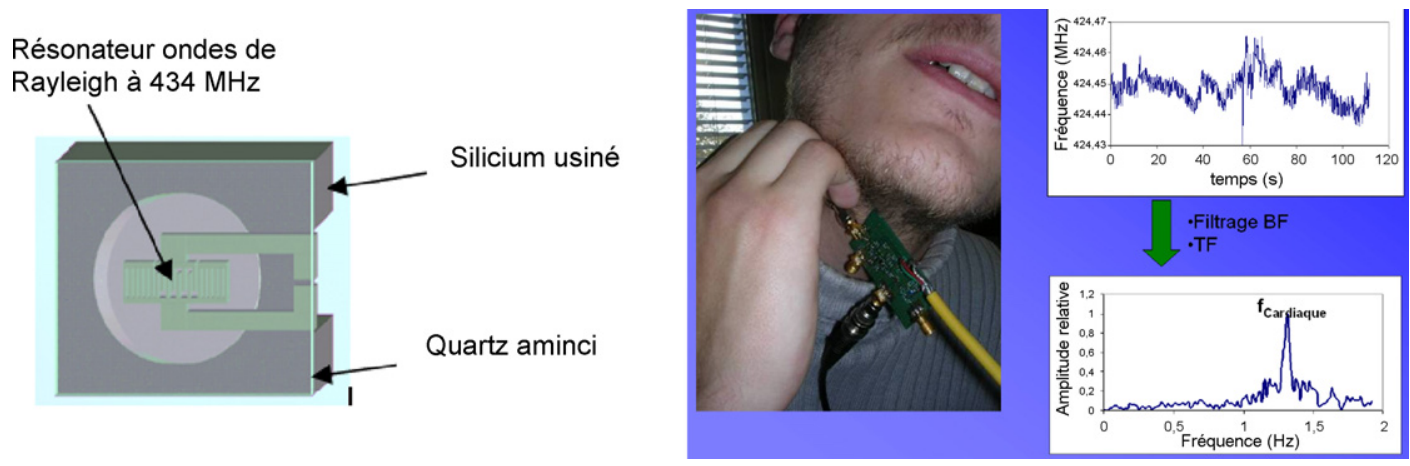

Fig. 11. Capteur de pression à $434 \mathrm{MHz}$ et résultats de mesure in vivo. 
sur une tranche de silicium usiné. L'ensemble est alors aminci côté quartz de façon à obtenir la sensibilité à la pression voulue (Fig. 11). Pour pallier les difficultés liées aux mesures in vivo à $2,45 \mathrm{GHz}$, des mesures de pression artérielle ex vivo (à la surface de la peau) par une méthode de mesure oscillométrique (mode filaire) ont été effectuées à l'aide de ce dispositif. Les variations de pression mesurées au niveau de l'artère brachiale sont traduites sous forme de décalage de fréquence permettant par calcul d'identifier les pressions diastoliques et systoliques. Les algorithmes de détection ainsi développés montrent un bon accord avec des mesures cliniques et celles effectuées au laboratoire.

\section{Conclusion et perspectives de développement}

Nous confirmons que les pertes de propagation à $2,45 \mathrm{GHz}$ dans l'air et dans les milieux biologiques ne permettent pas d'obtenir un niveau de signal compatible de la sensibilité du système d'interrogation. La réponse d'un résonateur SAW à $2,45 \mathrm{GHz}$ étant environ 30 fois plus courte que celle d'un résonateur à $434 \mathrm{MHz}$, la fréquence d'échantillonnage du convertisseur $\mathrm{A} / \mathrm{N}$ ne permet pas d'effectuer une numérisation correcte du signal. De surcroît les décharges parasites de l'électronique sont superposées temporellement avec la réponse du résonateur. Les essais de mesure de pression (dans la colonne d'eau en particulier) effectués en mode filaire sur des dispositifs de test type $\mathrm{P}$ ont montré que la fréquence de résonance était extrêmement sensible aux éléments parasites associés à l'environnement de mesure (capacité parasite liée à la présence du milieu aqueux). Cette sensibilité de la fréquence de résonance masque la variation de fréquence liée à la variation de pression.

En résumé la solution à $2,45 \mathrm{GHz}$ ne peut pas répondre au cahier des charges de l'application. Les premières mesures effec- tuées à $434 \mathrm{MHz}$ démontrent qu'il est possible de mesurer des variations de pression relatives avec une précision compatible de l'application. Nous préconisons de poursuivre ce développement (capteur implantable) à plus basse fréquence $(434 \mathrm{MHz})$ où les problèmes mentionnés ci-dessus sont minimisés. Les principaux obstacles que nous devrons surmonter sont la réduction de taille de l'antenne de façon à conserver le concept d'antenne intégrée sur quartz à cette fréquence. CIMPA nous a par ailleurs permis de développer toutes les briques technologiques qui seront utilisées pour la fabrication du capteur à $434 \mathrm{MHz}$.

\section{Conflit d'intérêt}

Aucun.

\section{Références}

[1] Reindl L, Scholl G, Ostertag T, Scherr H, Schmidt F. Theory and application of passive SAW radio transponders as sensors", IEEE transactions on ultrasonics. Ferroelectrics and Frequency Control 1998;45(5):1281-91.

[2] Buff W, Klett S, Rusko M, Ehrenpfordt J, Goroll M. Passive remote sensing for temperature and pressure using SAW resonator", IEEE transactions on ultrasonics. Ferroelectrics and Frequency Control 1998;45(5):1388-92.

[3] Ballandras S. Precise modeling of complex SAW structures using a perturbation method hybridized with a finite element analysis. In: IEEE Trans Ultrason. Symp. 1998.

[4] Collin G, Chami A, Luxey C, Le Thuc P, Staraj R. Human implanted spiral antenna for a $2.45 \mathrm{GHz}$ wireless temperature and pressure SAWsensor system", IEEE antennas and Propagation Society International Symposium, 2008. AP-S, 2008.

[5] Martin G, Berthelot P, Masson J, Daniau W, Blondeau-Patissier V, Guichardaz B, et al. Measuring in the inner body temperature using a wireless temperature SAW-sensor-based system. In: IEEE Ultrasonics symposium. 2005.

[6] Hermelin D, Daniaux W, Ballandras S, Belgacem B. Fabrication of surface acoustic wave wireless pressure sensor. In: IEEE IFCS. 2008 